\title{
Magnetic stimulation over the spinal enlargements
}

\author{
Y UGAWA, J C ROTHWELL, B L DAY, P D THOMPSON, C D MARSDEN \\ From the MRC Human Movement and Balance Unit, Institute of Neurology, London, UK
}

SUMMARY Magnetic stimulation over the cervical and lumbar spinal enlargements was performed in 10 normal volunteers using a $9 \mathrm{~cm}$ diameter coil. Although the threshold and the amplitude of responses depended on the position of the coil and the direction of current flow within it, the latency was constant. The latencies obtained by magnetic stimulation were compatible with those obtained using high voltage electrical stimulation of the spinal nerve roots and always were shorter than the peripheral motor conduction time estimated by $\mathrm{F}$-wave techniques. The site of activation by magnetic stimulation appears to be very similar to that stimulated by the high-voltage electrical method. Stimulation of descending motor tracts within the cord was not possible using the magnetic stimulator.

It has been known for many years that rapidly changing magnetic fields are capable of stimulating nervous tissue.' Since the discovery that these methods can be used to activate the human brain through the scalp without causing any discomfort, ${ }^{2}$ the use of the technique has expanded considerably. The most common clinical application of brain stimulation, like that of sensory evoked potential methods, has been to measure the conduction time in central motor pathways. These estimates are made by measuring the latency of EMG responses in active muscles to stimulation over central areas of scalp, and subtracting from that value the time consumed by the impulses travelling in peripheral motor pathways from spinal cord to muscle. ${ }^{34}$ The latter can be estimated from Fwave measurements, or by using high-voltage stimulation over the spinal enlargements. The action of direct electrical stimulation over the spinal column has been investigated by several authors. At cervical levels, it has been suggested that peripheral motor axons are likely to be activated about $4 \mathrm{~cm}$ distant to the anterior horn cells. ${ }^{5}$ In the lumbo-sacral region, activation may occur at two sites: within the cauda equina, or at the exit of the motor roots from the spinal column. ${ }^{6}$ Stimulation of such proximal sites of the motor axons provides a direct and non-invasive way of assessing conduction in virtually all of the peripheral motor pathway.

Address for reprint requests: Dr J C Rothwell, MRC Human Movement and Balance Unit, Institute of Neurology, Queen Square, London WCIN 3BG, UK.

Received 29 November 1988 and in revised form 16 March 1989. Accepted 22 March 1989
The purpose of the present paper is to show that the presently available magnetic stimulators are also capable of stimulating motor axons of peripheral nerve near their exit from the spinal column. The site of stimulation appears to be very similar to that activated by the high-voltage electrical method, although the threshold at which effects occur depends critically on the position of the coil and the direction of current flow within it.

\section{Subjects and methods}

Ten normal volunteers (seven male, three female: age range 27-35 years) including the authors were studied. All gave informed consent; the procedures were approved by the local ethical committee.

Recordings were made from the following muscles: biceps brachii, thenar, quadriceps femoris, tibialis anterior (TA), gastrocnemius and extensor digitorum brevis (EDB). The electromyographic (EMG) response was recorded with surface $\mathrm{Ag} / \mathrm{AgCl}$ electrodes fixed 2-3 cm apart over the muscles. Signals were amplified with Devices 3160 preamplifiers with filters set at $80 \mathrm{~Hz}$ and $2.5 \mathrm{kHz}$ and then by a Devices 3120 amplifiers. Data were collected and stored on floppy disc by a PDP 12 computer. The latency and peak-to-peak size of the muscle action potentials were measured by eye on the computer visual display unit.

We used a prototype of the Novametrix Magstim 200 magnetic stimulator built by Dr A Barker and colleagues of the Medical Engineering Department of Sheffield University. $^{2}$ A pulsed magnetic field (2.1T max at $\left.230 \mu \mathrm{s}\right)$ was applied via a $9 \mathrm{~cm}$ mean diameter flat coil. The centre of the coil was placed over the spinous processes at various levels: the second cervical (C2) to the fourth thoracic (Th4) spinous process for the upper limb muscles, and the twelfth thoracic (Th12) to the second sacral (S2) spinous processes for the lower limb muscles. The intensities of stimulation were 
increased stepwise from $30 \%$ to $100 \%$ of the maximum output of the device. Both clockwise and counter-clockwise current flow in the coil were used. At least three responses were recorded from each muscle for each stimulation condition (site, intensity and current direction in the coil). Percutaneous electrical stimulation over the vertebral column was also performed using a prototype of the commercially available Digitimer D 180 built by $\mathrm{Mr}$ H B Morton. ${ }^{7}$ This device had a maximal output of $750 \mathrm{~V}$ and a decay constant of $50 \mu \mathrm{s}$. Stimuli were delivered through $\mathrm{Ag} /$ $\mathrm{AgCl}$ cup electrodes taped to the skin. We used monopolar stimulation similar to that previously described. ${ }^{6}$ The cathode was placed over the spinal column (at C5, C7, L2, L4, S1, and $\mathrm{S} 1$ spinous processes for biceps, thenar, quadriceps, TA, gastrocnemius and EDB muscles respectively) and the anode over the shoulder or iliac crest contralateral to the limb being examined. With this montage the responses are thought to be evoked by the stimulation of the motor roots at their exit from the spinal canal. ${ }^{568}$ As with magnetic stimulation, intensity is expressed as a percentage of the maximum output of the device. Stimulation of the cervical cord was performed with the subjects seated comfortably in a reclining chair; subjects lay prone during lumbar stimulation.

Latencies obtained by magnetic stimulation in thenar, TA and EDB muscles were compared with peripheral latencies calculated using conventional F-wave method. At least 20 successive $F$-waves were recorded from these muscles to define the shortest F-wave latency. Total peripheral motor conduction time was calculated from the formula $(F+M-$ 1) $/ 2$, where $F=$ latency of $F$-wave: $M=$ latency of $M$-wave. This formula is that given by Kimura, ${ }^{9}$ in which $1 \mathrm{~ms}$ is subtracted from the F-wave latency in order to account for the turn-around time of the antidromic volley at the motoneuron. This "corrected" F-latency is therefore equal to the time taken for a volley to travel from the point of stimulation to the spinal cord and then back to the muscle. Adding on the M-wave latency, this becomes equal to twice the motor conduction time from spinal cord to muscle.

\section{Results}

Consistent motor responses could be obtained in all muscles in all subjects. No unwanted side effects of the stimulation were observed.

\section{(1) Magnetic stimulation over the cervical column}

The threshold and amplitude of the EMG responses was markedly influenced by the direction of current flow in the coil. Figure 1 shows responses recorded in the left thenar muscle at different intensities of stimulation when the centre of the coil was placed over the fifth cervical (C5) spinous process. In this position, the exits of the motor roots innervating the thenar muscles ( $\mathrm{C} 8$ and $\mathrm{T} 1)$ were then under the lower horizontal part of the coil. When the current in the stimulating coil flowed counter-clockwise, the responses were larger and the threshold lower than when clockwise stimulation was given. Figure $2 \mathrm{~A}$ shows representative motor action potentials from the right thenar muscle of a normal subject after $70 \%$ stimula-

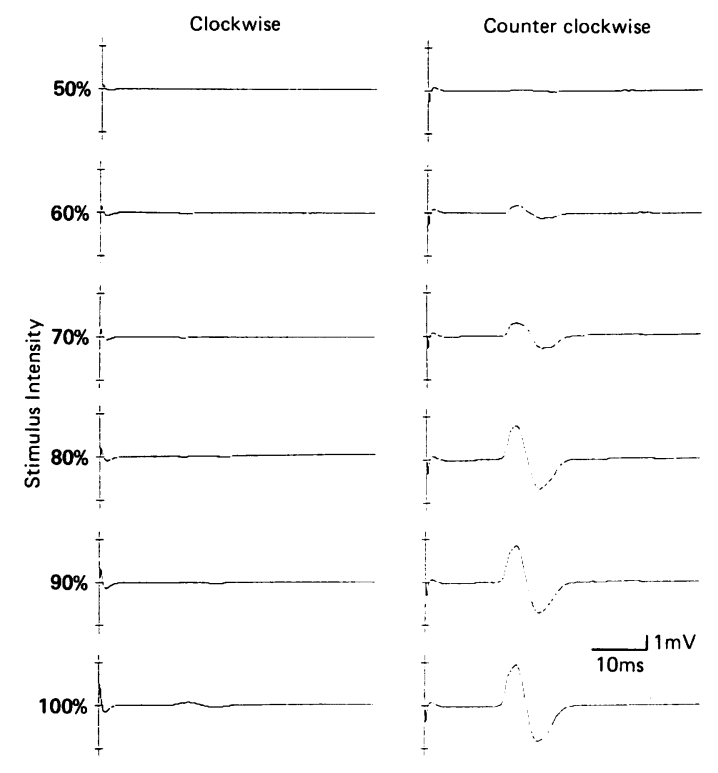

Fig 1 Responses (average of three trials each) in the left thenar muscle of a typical normal subject to different intensities of magnetic stimulation with the coil centred over the fifth cervical spinous process. The threshold is lower, and the responses larger when counter-clockwise stimulation is used.

tion with the magnetic coil centred at various levels of the cervical and upper thoracic cord. Figure 2B shows the mean peak-to-peak amplitudes of thenar responses from six subjects at these different levels. Maximum responses could be obtained with the coil centred over C5 spinous processes if the stimulating current flowed clockwise. In contrast, counter-clockwise stimulation produced the largest responses when the coil was centred over T3. The exits of the motor roots innervating the thenar muscles were under the lower horizontal part of the coil when the centre of the coil was placed over the $\mathrm{C} 5$ spinous process and they were under the upper horizontal part of the coil when placed over the T3 spinous process. Therefore, the response in the right thenar muscles was largest and the threshold was lowest when (1) the exit of the motor roots at $\mathrm{C} 8$ and T1 were under the horizontal part of the coil and (2) the current flow at this part of the coil was from the muscle towards the spinal cord. To obtain the largest responses in the left side the flow of the current had to be reversed. The size ratio between the largest responses to magnetic stimuli and maximal $M$-waves in the thenar muscle produced by median nerve stimulation at the wrist was quite variable among subjects and ranged from $16-77 \%$. In seven subjects we also examined the best location for obtaining responses in 

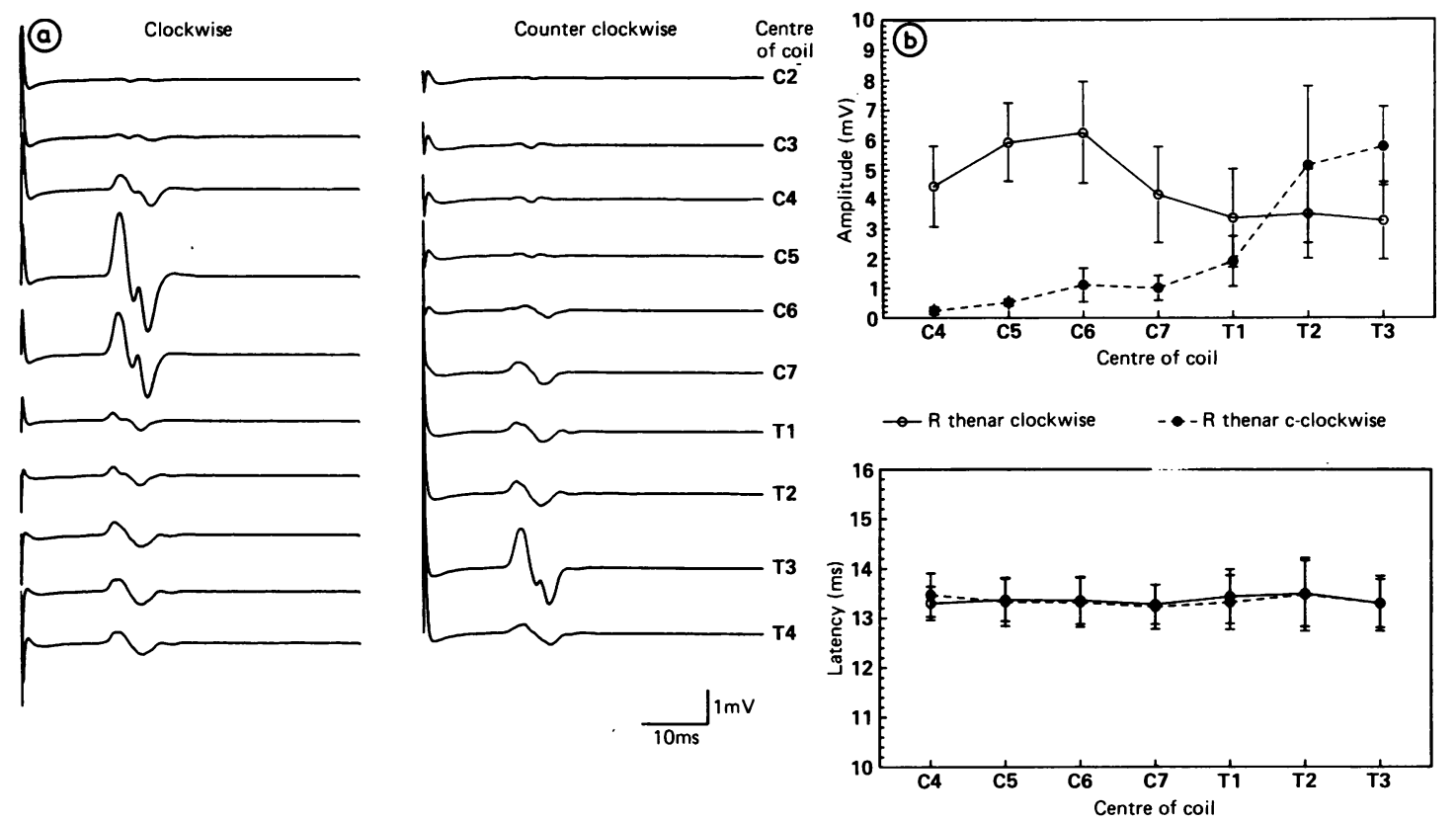

$\rightarrow-R$ thenar clockwise $\quad-\bullet-R$ thenar c-clockwise

Fig 2 (A) Responses (average of five trials each) in the right thenar muscle evoked by $70 \%$ stimulation with the magnetic coil centred at various levels of the cervical cord. Maximum responses are provoked when the coil is centred over C5 spinous process if the stimulating current flows clockwise. When the current is reversed (counter-clockwise stimulation) responses are largest at T3. The response latencies are approximately constant. (B) Average (1 SE) peak-to-peak amplitudes of the maximum thenar $M$-waves in six subjects after magnetic stimulation over the cervical column at different levels. (C) Average (ISE) latencies to onset of thenar muscle action potentials in the same subjects.

the biceps muscle. The principle was the same as for the thenar muscles. Responses were largest and of lowest threshold when the horizontal edge of the coil was over the exit of the motor roots at C5 and C6, so that the current in it flowed in the direction from muscle to cord.

It should be noted that although stimulation was most effective if the coil was centred at a particular location, smaller responses could be obtained over several segments of the cord. The threshold and amplitude of these EMG responses varied depending on the direction of the current flow and the intensity of stimulation but the latency of the response was constant (figs 1, 2C).

In contrast to the known effects of electrical stimulation over spinal cord ${ }^{5}$ magnetic stimulation over the cervical cord never elicited any responses in the leg even when maximum intensities of stimulation were used.

(2) Magnetic stimulation over the lumbosacral column As for cervical stimulation, large responses could be obtained when the horizontal edge of the coil was located over the innervating motor roots and current in the coil flowed in the direction from the muscle towards the spine. However, the size of responses in leg muscles was less sensitive to direction of current flow in the coil.

For right quadriceps muscle (see fig 3A), clockwise stimulation produced the largest responses when the coil was centred over $\mathrm{Ll}$, that is, the lower horizontal part of the coil was located over the point of exit of the motor roots innervating quadriceps (namely L3 and L4). Responses became smaller when the centre of the coil was placed lower over the emerging motor roots of the L3/4 level of the spinal column. If the coil was moved further caudally with its centre over L5, S1 or S2, large responses were elicited again. With counterclockwise stimulation responses first became large with the coil centre over L5, S1 and S2, when the upper horizontal part of the coil was located over the motor roots at L3 and L4. Similar responses were observed in all subjects (see fig 3B). As with the thenar muscles, the latency of responses was similar at all levels of stimulation (fig 3C).

Responses in TA, gastrocnemius, and EDB were 

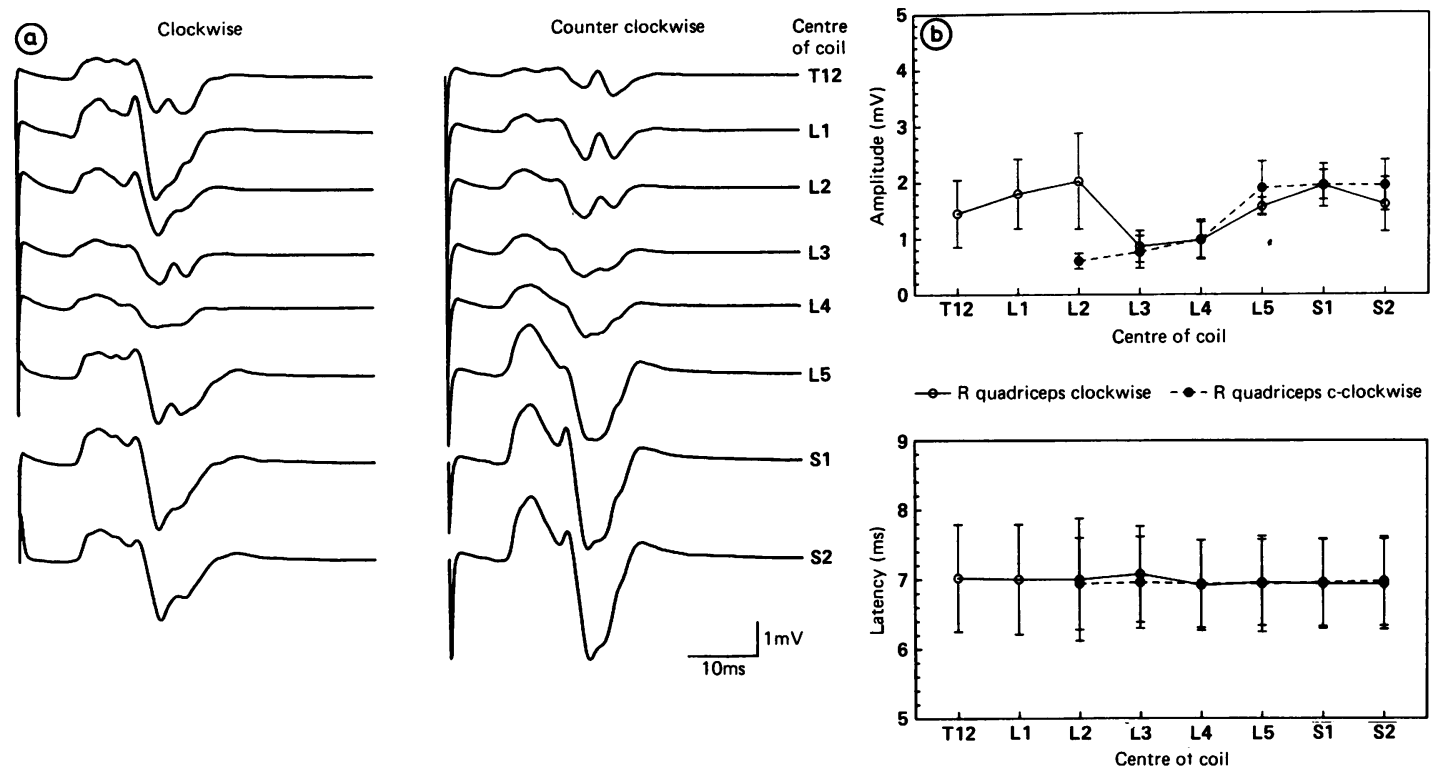

$\rightarrow R$ quadriceps clockwise $-\bullet-R$ quadriceps c-clockwise

Fig 3 (A) Responses (average of three trials each) in the right quadriceps femoris muscle evoked by 100\% stimulation at various levels of lumbosacral cord. With clockwise stimulation, the response at L1 is larger than those immediately below or above that level. They become large again as the coil is moved caudally to lie with its centre over L5, S1 or S2. With counterclockwise stimulation, responses are small over high lumbar levels, and become larger as the upper part of the coil lies over the motor roots (that is when the centre is over L5). The responses continue to be large as the coil is moved caudal to this point. Latencies are approximately constant. $(B)$ and $(C)$ average (1 SE) peak-to-peak amplitudes (B) and onset latencies (C) of maximal $M$-waves elicited in the quadriceps muscle of five subjects after magnetic stimulation over different levels of lumbosacral cord.

slightly different from those in quadriceps because of the lower level at which their motor roots exit from the spinal column. Figure 4A illustrates typical responses in the right EDB muscle when magnetic stimulation at $100 \%$ was given with the coil centred at various levels in the lumbosacral region. Mean results from all subjects are shown in fig 4B. With clockwise stimulation, responses became large with the coil centred over L4/L5, that is, with the lower horizontal part of the coil over the exits of the motor roots innervating EDB (namely at L5 and S1). However, unlike the situation in the cervical cord, or in quadriceps, the responses did not decrease in size as the coil centre was moved over S1 and S2. In fact responses were larger with the coil centred at S2 than at L4. In these muscles, clockwise stimulation always produced larger responses in the right leg than counter-clockwise stimulation. As in the thenar muscles, the ratio between the size of the responses to magnetic stimuli over the lumbar column and the maximal peripheral nerve $\mathbf{M}$-wave was again variable. In TA and EDB it ranged from $10-90 \%$ and $15-50 \%$ respectively.
The latency of EMG responses was constant if their amplitude was greater than $1 \mathrm{mV}$ (fig 4C).

\section{(3) Comparison with percutaneous electrical stimula- tion}

The observation that the response latencies were constant in both the upper and lower limb muscles, despite changes in coil position, suggests that activation always occurred at the same site. These latencies were compared with those obtained by percutaneous electrical stimulation. It should be noted that, as described in Methods, electrical stimulation was applied in such a way as to activate preferentially the spinal nerve roots near their exit from the spinal column. Descending fibre tracts within the cord probably were not activated, nor, in the lumbar region, was the cauda equina activated. ${ }^{6}$ The latencies obtained by magnetic stimulation versus those obtained by percutaneous electrical stimulation in the same muscle are shown in fig 5, A, B, C. There were no significant differences between magnetic and electrical stimulation of the motor roots in thenar, quadriceps 

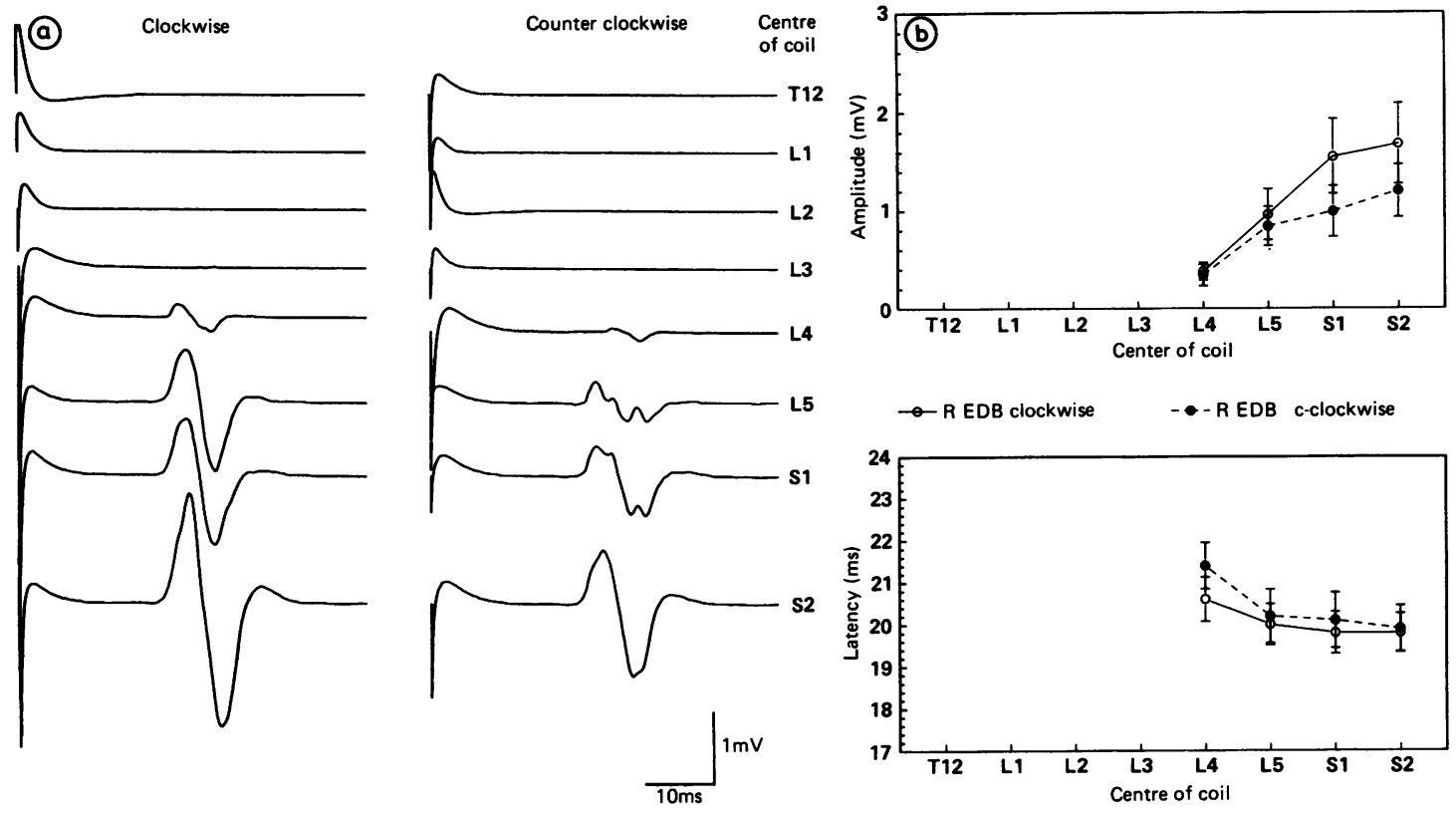

○ OR EDB clockwise $\quad--\cap$ R EDB c-clockwise

Fig 4 (A) Responses (average of three trials each) in the right EDB evoked by $100 \%$ stimulation at various levels of lumbosacral cord. Large responses can be obtained at L5 or more caudal levels with both orientations of the coil. They are larger using clockwise stimulating current than those using counter-clockwise current. Latencies are approxmately constant. (B and $C$ ) average (1 SE) peak-to-peak amplitudes (B) and onset latencies (C) of maximal $M$-waves elicited in the EDB muscle of six subjects after stimulation over different levels of lumbosacral cord.

femoris, TA, gastrocnemius, and EDB muscles (paired $t$ test, $\mathrm{p}>0.05$ ). However, the latency to electrical stimulation was slightly shorter in biceps brachii (mean difference: $0.11 \mathrm{~ms}, \mathrm{p}<0.05$ ).

\section{(4) Comparisons with F-wave latencies}

Latencies of the responses in thenar, TA and EDB to magnetic stimulation were compared with conventional estimates of peripheral motor conduction times using the F-wave techniques (fig 6). The latencies were always shorter than $(F+M-1) / 2$. The mean differences were $1.4,3.0$ and $3.5 \mathrm{~ms}$ in the thenar, TA and EDB, respectively.

\section{Discussion}

The present investigation has shown that: (1) During magnetic stimulation over the cervical column, the amplitude of the muscle response is largest and the threshold is lowest when the horizontal part of the coil is over the exit of the motor roots and the current flow in the coil is directed from the muscle to the spinal cord at this point. The situation is similar for the lum- bosacral column, except that the effect of direction of current flow in the coil is not so dramatic, (2) The latencies of the responses are constant for both cervical and lumbosacral stimulation despite moving the coil centre several segments away from the lowest threshold point, (3) The latencies obtained by magnetic stimulation are the same as those of electrical stimulation and they are always shorter than $(F+M$ $-1) / 2$.

The effects at cervical level probably can be explained as follows. The current induced in the conductive tissues of the body flows in a direction opposite to that in the coil. Thus, when the horizontal part of the coil is placed over the exits of the nerve roots with the coil current flowing in the direction from muscle to cord, the induced current probably flows along the pathway of the nerve trunk from cord to muscle. The effect may be similar to stimulating with a distal cathode and a proximal anode. If the coil current is in the opposite direction (equivalent to a distal anode), the stimulus is less effective although responses may still be obtained. If the coil is centred over the root exit levels, the vertical edge of the coil 
passes over the motor nerves. In this case, the induced current in the tissues will flow at right angles to the nerve trunk, and the threshold will be relatively high.
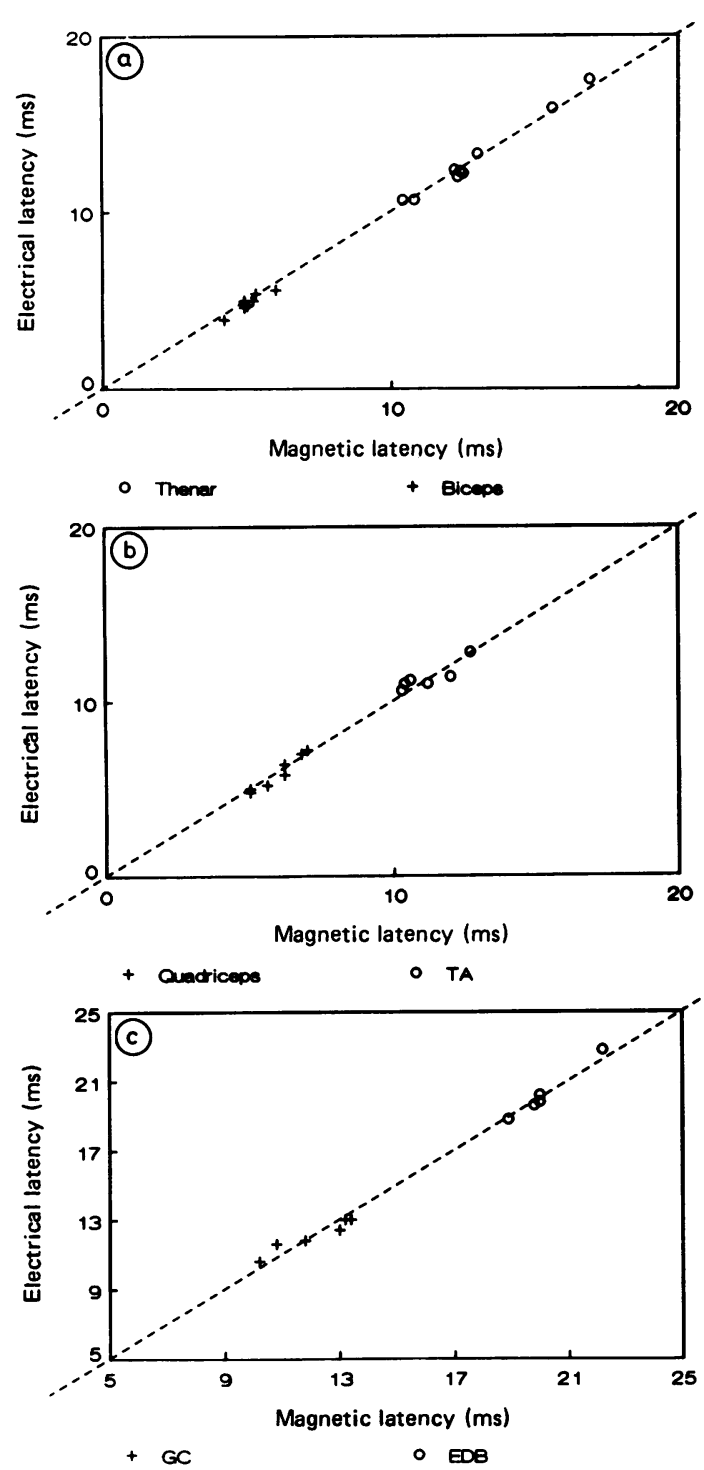

Fig 5 Latency comparisons between magnetic and high voltage electrical stimulation over the cervical or lumbosacral column in thenar and biceps (top), quadriceps and tibialis anterior (TA) (middle), and gastrocnemius (GC) and EDB (bottom). Latencies to the two forms of stimulation are the same in all muscles except biceps, where electrical latencies are slightly shorter $(0.11 \mathrm{~ms})$ than magnetic. The angled dotted line is the line of identity. For each muscle; each point represents results from a single individual.

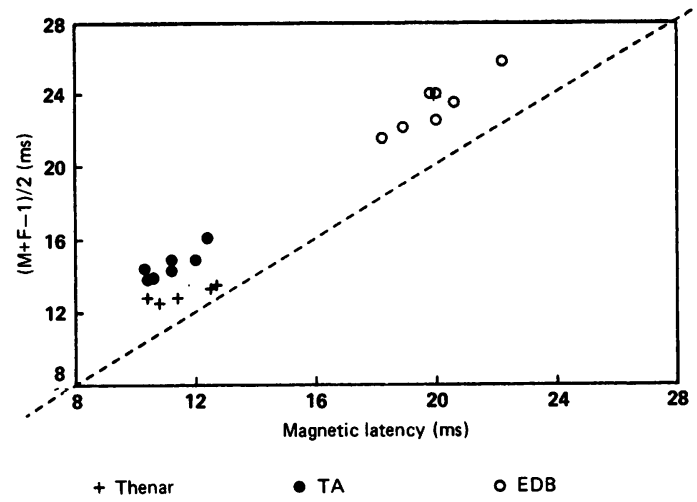

Fig 6 Comparison between magnetic latencies obtained from cervical or lumbosacral cord stimulation with total peripheral motor conduction times obtained by the $F$-wave technique. Results shown from thenar, TA and EDB muscles.Magnetic latencies always are shorter than $(M+F-1) / 2$. The angled dotted line is the line of identity. For each muscle, each point represents results from a single individual.

At the lumbosacral level, responses in quadriceps behaved similarly to those at cervical levels. Large responses were seen when the horizontal part of the coil was over the exit point of the innervating motor roots, and smaller responses were seen when the vertical edge was at right angles to the exit point of the roots. The effect of stimulus direction was less dramatic than in the arm. In TA, gastrocnemius, and EDB, the situation was slightly different. As the coil was moved caudally, responses first became large when the lower horizontal part of the coil lay over the exit of the motor roots from the spinal column. As at cervical levels, responses in the muscles of the right leg were larger when clockwise stimulation was used (that is when the current in the coil flowed in the direction from muscle to cord). However, the responses did not become smaller as the coil centre was moved downwards over the root exits; indeed they became bigger.

One reason for this may be because the orientation of the roots making up the sciatic nerve is different from those at cervical levels. At the cervical level motor nerve roots course laterally from the spinal cord and continue in this direction after leaving the spinal canal through the intervertebral foramina until they unite to form the brachial plexus. In contrast, roots of the sciatic nerve have a significant longitudinal component both as they course through the cauda equina, and following their exit from the spinal canal where they course vertically to form the sacral plexus. Since the fibres also have a relatively horizontal course through the intervertebral foramina they may be stimulated effectively by both vertical and horizontal 
components of induced current flow. Thus, as the stimulating coil is moved down the column, the lower horizontal edge will first lie over the site at which the motor roots exit the column. At this point the horizontal component of current flow may cause current to flow along horizontal sections of nerve. Moving the coil lower, its vertical edge may then begin to induce current to flow along vertically-oriented sections of nerve. We cannot say precisely at what point activation actually occurs with either orientation of current flow (see below). However, since the latencies to muscle activity are equal with both locations of the stimulating coil, the two points are likely to be the same, or at least very close to one another. Placement of the coil well below the root exit zone was not possible for these muscles because of the short length of the sacral column in man.

A surprising feature of the motor responses is their constant latency. This implies that there is some low threshold point on the nerve which is preferentially activated by applied current, even when the stimulating coil is placed well away from the point of lowest threshold. From latency measurements, this point appears to be the same point as that activated by high voltage electrical stimulation, at least over the cervical column. Electrical stimulation over the lumbar column can activate motor nerves at several sites. ${ }^{6}$ In the present experiments, magnetic stimulation produced responses at latencies which correspond to electrical stimulation of the roots near to their exit from the spinal column. We do not know the precise point of stimulation either at lumbar or cervical levels. At cervical levels, Mills and Murray ${ }^{5}$ have compared latencies after electrical stimulation with those obtained from F-wave estimates. They concluded that activation probably occurs at about $4 \mathrm{~cm}$ distal to the spinal motoneurons themselves. However, estimation of the point of stimulation depends on three variables. (1) Whether the F-wave estimates the speed of conduction in the most rapidly conducting fibres, and if so, the degree to which conduction of the orthodromic impulse in these fibres may be slowed by the passage of the preceding antidromic volley. This will be particularly important in the most proximal part of the nerve where the antidromic and orthodromic volleys are separated by only $1-2 \mathrm{~ms}$. (2) The turn-round time of the F-wave at the motoneurone cell body and (3) the value taken for the conduction velocity in the most proximal portion of the motor axon. There is virtually no information on any of these factors in man, so that any estimate will be unreliable. For example, when Mills and Murray ${ }^{5}$ compared the peripheral conduction times to the hypothenar muscles using measurements based on $\mathrm{F}$-wave techniques with that obtained by electrical stimulation over the cervical enlargement, they found a latency difference of $0.6 \mathrm{~ms}$. In the present results, we arrive at a value of $1.4 \mathrm{~ms}$ for the thenar muscles. The reason for the difference is that Mills and Murray use the formula $(\mathrm{M}+\mathrm{F}) / 2-1$ to calculate total peripheral conduction times, whereas we have used $(M+F-1) / 2$ (see Methods). At the present time there is no way of knowing which is correct, even though a time difference of $0.8 \mathrm{~ms}$ in fibres conducting at $60 \mathrm{~ms}^{-1}$ corresponds to a distance of $48 \mathrm{~mm}$.

Unlike electrical stimulation, with the magnetic coil we were unable to stimulate the fibre tracts within the cord itself. The reason for this has been described by Dr A Barker and colleagues. ${ }^{10}$ The current induced by the magnetic coil is theoretically maximum in the annulus under the coil. Because the bony vertebrae act as an insulator between the spinal cord and the external tissue, current probably tends to flow around the spinal column rather than through it. Some current will be induced in the conductive tissue within the bony canal, but because the volume of the tissue is small, this current will be correspondingly low.

The size ratio between the largest responses to magnetic stimuli over the cord and maximal peripheral nerve $\mathbf{M}$-waves was very variable, ranging between $10-90 \%$. This means that magnetic stimulation cannot be relied upon to produce supramaximal effects, at least when given over the spinal enlargements. Thus, in contrast to percutaneous high voltage stimulation over the cervical cord or direct stimulation of spinal nerve roots with needle electrodes which always give supramaximal effects, magnetic stimulation is likely to be of little use in documenting conduction block in proximal peripheral nerves.

In conclusion, magnetic stimulation over the spinal enlargements is well tolerated and relatively painless. The latency measurements appear compatible with those obtained using electrical stimulation and are relatively insensitive to changes in coil position as long as the size of the EMG response is $>1 \mathrm{mV}$. To obtain responses of lowest threshold, at least at cervical levels, the coil should be placed so that the horizontal edge lies over the exit of the motor roots from the spinal column, and the current in the coil should flow in the direction from muscle to cord.

We thank Dr A Barker and colleagues for constructing the magnetic stimulator used in these experiments; $\mathbf{M r}$ H B Morton who kindly gave us the prototype electrical stimulator; Dr $\mathrm{K}$ Inoue (Department of Neurology, University of Tokyo) who kindly helped us with his expert knowledge on spinal root anatomy. Mr R Bedlington was, as usual, indispensible. The work was funded by the Medical Research Council and the British Council. 


\section{References}

1 Thompson SP. A physiological effect of an alternating magnetic field. Proc $R$ Soc Ser B 1910;82:396-8.

2 Barker AT, Jalinous R, Freeston IL. Non-invasive magnetic stimulation of the human motor cortex. Lancet 1985;1:1106-7.

3 Merton PA, Morton HB, Hill DK, Marsden CD. Scope of a technique for electrical stimulation of human brain, spinal cord, and muscle. Lancet 1982;ii:597-600.

4 Barker AT, Jalinous R, Freeston IL, Jarratt JA. Clinical evaluation of conduction time measurements in central motor pathways using magnetic stimulation of human brain. Lancet 1986;1:1325-6.

5 Mills KR, Murray NMF. Electrical stimulation over the human vertebral column: which neural elements are excited? Electroencephalogr Clin Neurophysiol 1986;63:582-9.

6 Maertens de Noordhout A, Rothwell JC, Thompson PD,
Day BL, Marsden CD. Percutaneous electrical stimulation of lumbosacral roots in man. $J$ Neurol Neurosurg Psychiatry 1988;51:174-81.

7 Merton PA, Morton HB. Stimulation of the cerebral cortex in the intact human subject. Nature 1980;285:227.

8 Ugawa Y, Genba K, Shimpo T, Mannen T. Physiologic analysis of central motor pathways-simultaneous recording from multiple relaxed muscles. Eur Neurol 1989;29:135-40.

9 Kimura J. F-wave velocity in the central segment of the median and ulnar nerves: a study in normal subjects and patients with Charcot-Marie-Tooth disease Neurology 1974;24:539-46.

10 Barker AT, Freeston IL, Jalinous R, Jarratt JA. Magnetic stimulation of the human nervous system: an introduction and principles. In: Chokroverty S, ed. Magnetic Stimulation in Clinical Neurophysiology. London: Butterworths (in press). 